\title{
Seasonal skin darkening in Chinese women: the Shanghaiese experience of daily sun protection
}

This article was published in the following Dove Press journal:

Clinical, Cosmetic and Investigational Dermatology

30 May 2013

Number of times this article has been viewed

\author{
Huixia Qiu' \\ Frederic Flament ${ }^{2}$ \\ Xiaohui Long' \\ Jun $\mathrm{Wu}^{\prime}$ \\ Mengzhi $\mathrm{Xu}$ \\ Didier Saint Leger ${ }^{2}$ \\ Helene Meaudre ${ }^{2}$ \\ Jerome Senee' \\ Bertrand Piot ${ }^{2}$ \\ Roland Bazin² \\ 'L'Oréal Research and Innovation, \\ Shanghai, People's Republic of China \\ 'L'Oréal Research and Innovation, \\ Chevilly, France
}

Correspondence: Huixia Qiu L'Oréal Research Shanghai, 550 jin Yu Road, Jinqiao, Pudong Area, 201206, Shanghai, People's Republic of China

Tel +862138723785

Fax +86 21 3872372

Email cqiu@rd.loreal.com

\begin{abstract}
The facial skin tone of two groups of Chinese women from Shanghai was compared using standard colorimetric space techniques during a 6-month interval between January and July 2011. During the study period, one group of women $(n=40)$ applied a potent sun-protective cosmetic product daily, while the other group $(n=40)$ did not use any sun protection. The results, based on images taken using a standardized digital camera coupled to a spectroradiometer, showed that sun protection largely mitigated changes in the components of skin tone, ie, lightness, melanization, and individual typology angle parameters. The skin darkening process appeared to be reduced or prevented in the sun-protected group when compared with the control group. The sun-protected women had participated in an earlier study in 2008, which confirmed that seasonal skin darkening occurs from winter through summer in Shanghaiese women. Comparing the data obtained in the winters of 2008 and 2011, we were able to identify better the impact of 3 years of aging on the components of skin tone. Comparing data between seasons on the same women with (2011 study) and without (2008 study) sun protection highlights the role of the test product in preventing skin darkening.
\end{abstract}

Keywords: skin tone, skin seasonal darkening, sun protection, aging

\section{Introduction}

For decades, Western culture has attached much esthetic value to skin tanning because of its association with apparent good health, playing sport, and taking vacations. However, as early as the 1930 s, Coco Chanel, a pioneer in cosmetics, warned about the likely long-term negative impact of sun exposure on skin aging. Since then, a large body of research has confirmed this negative effect. ${ }^{1,2}$ In contrast with the West, skin darkness is perceived negatively, both individually and socially, throughout Asia, including in China, where it is often associated with strenuous outdoor activities, such as farming and manual labor, with a fair skin suggesting a higher social rank. In Chinese culture, the association between skin darkening and sun exposure has been known for centuries, accounting for the traditional use of sun-protection aids such as the umbrellas and face shields used by women on sunny days. Fear of skin darkening largely accounts for the skin whitening products in vogue throughout Asia.

Climatic conditions in Shanghai are such that the city has relatively short springs and autumns, with summer rapidly giving way to winter and vice versa, which enabled us to focus on the possible structural and functional changes occurring in the skin of 354 Shanghaiese women aged 18-80 years during these two successive periods. ${ }^{3,4}$ Signs of aging and clinical features were shown to be unaltered over a 6-month period, 
whereas some functional criteria appeared to be affected, in particular skin pigmentation. The main results were a steady age-related decrease in $\mathrm{L}^{*}$ (skin lightness), with a slope of about 1.0 unit per decade. The results also showed that, from winter to summer, almost all parameters of skin pigmentation were significantly affected, ie, $L^{*}$ decreased, and the number and surface area of hyperpigmented spots increased, as well as their "melanin index" recorded using a Mexameter" (Courage Khazaka Electronic GmbH, Cologne, Germany). ${ }^{3}$ In most cases, these changes did not seem to be particularly affected by aging during the 6-month period for subjects of any age. Based on these observations, we propose referring to this phenomenon as "seasonal skin darkening".

The association between seasonal skin darkening and the natural increase in daylight and ultraviolet irradiance from winter through summer led us to evaluate the possible effect of a commercial whitening product on a subgroup $(n=38)$ of this cohort of women over a 2-month period (from January to March). ${ }^{4}$ The tested product contained both a whitening active ingredient and sunscreens of a SPF18 and PA+++ protection level, which was applied to facial skin once daily. Women using this product showed a significant mean increase in $\mathrm{L}^{*}$ of +2.0 units and an increase in individual typology angle of +3.0 units, ie, increased skin lightness, which was noticed and recorded by almost all themselves on a self-administered questionnaire. However, the design of this study did not allow us to assess the extent to which an ultraviolet A and B sun protection product applied daily might prevent or at least mitigate seasonal skin darkening. The research presented here attempted to answer this question.

\section{Materials and methods Study design}

This 6-month study included two assessment sessions at our facilities at Pudong, Shanghai, one in January 2011 and one in July 2011, and assessed changes in facial skin tone in two age-matched cohorts of Chinese women with skin phototypes II-III. All subjects had been living in Shanghai for more than 15 years. One group $(n=40)$ was instructed to apply a high ultraviolet protection product (L'Oreal UV Perfect, SPF 50+, UVA protection factor 18) daily in the morning for 6 months. The test product was supplied in an unlabelled jar. The other group $(n=40)$ was instructed not to use any type of sun-protection product and served as a control. Both groups were allowed to continue their usual daily cleansing and moisturizing routine. All subjects attended our facility at the beginning and end of the study for automated photoimaging of their skin tone.

\section{Subjects}

The sun-protected group included 40 women aged 35-65 years who were selected from our previous 2008 study. ${ }^{3,4}$ These volunteers were recalled to meet our secondary aim of identifying any changes in facial skin tone over a 3-year period. The control group included 40 women recruited from various Shanghai agencies. All 80 women were informed about the conditions of the study and signed their informed consent. Women with skin disease and those on regular medical treatment were excluded from participation in the present study.

\section{Data recording}

Recordings were taken from the cheeks using a Chromasphere ${ }^{\circledR 5}$ device (Monaderm, Monaco) which includes a lighting system combined with an imaging system. The light (xenon lamp specification D65) used was stable and diffuse, and faithfully mimicked natural daylight. The image recording system consisted of both a tri-charged coupled device camera (Hitachi, Tokyo, Japan) and a spectroradiometer for data acquisition. The camera was calibrated to ensure reliable imaging with faithful color reproducibility. Quantitative data from measurements of skin complexion were expressed using the International Commission on Illumination (CIE) 1976 standard colorimetric values, ie, $\mathrm{L}^{*}, \mathrm{a}^{*}, \mathrm{~b}^{*}$.

We described skin tone quantitatively by recording: the $\mathrm{L}^{*}$ characteristic of lightness, whereby the higher the value of $\mathrm{L}^{*}$, the lighter the skin (black-white axis); $b^{*}$, the yellowblue component characteristic of melanin in yellow skin; $\mathrm{a}^{*}$, the red-green component; and the individual typological angle (ITA) that defines the degree of skin pigmentation by including lightness $\left(\mathrm{L}^{*}\right)$ and the melanization parameter $\left(\mathrm{b}^{*}\right)$, according to the following formula:

$$
\mathrm{ITA}=\left[\operatorname{Arctan}\left(\mathrm{L}^{*}-50\right) / \mathrm{b}^{*}\right] \times 180 / \pi
$$

where the higher the ITA value, the lighter the skin.

\section{Statistical analysis}

The Statistical Package for the Social Sciences version 16 (SPSS Inc, Chicago, IL) was used for the statistical calculations. The data are expressed as the mean \pm standard error unless otherwise stated. Statistical analysis was performed using the paired-samples Student's $t$-test for 
seasonal efficacy of the product and age-related skin changes in the same group. All tests were two-sided, and $P<0.05$ was considered to be statistically significant.

\section{Results}

\section{Effects of sun protection on skin tone}

Figures 1-3 illustrate the changes in $\mathrm{L}^{*}, \mathrm{~b}^{*}$, and ITA in both groups from January through July 2011. Two key findings emerge from these data. First, they are consistent with the findings of our earlier study in 2008 showing an approximately 1.0 unit decrease in skin lightness $\left(\mathrm{L}^{*}\right)$ from winter through summer in the control group (Figure 1), whereas the sun-protected group showed no such decrease. Parameter $b^{*}$, reflecting the yellow component of skin, increased from January through July in both groups, but to a lesser extent in the sun-protected group than in the control group (Figure 2). With regard to ITA, a more dynamic parameter which is mathematically influenced by an increase in $b^{*}$, applying a sun-protective cosmetic product clearly mitigated these changes, with the skin becoming slightly darker, with an approximate 0.5 unit decrease in ITA in the sun-protected group versus a 4.0 unit decrease in the control group (Figure 3). Overall, these results indicate a benefit on facial skin tone from daily sun protection in Chinese women (Figure 4). Figures 1-3 show that there was no statistically significant difference in the $L^{*}, b^{*}$ and ITA parameters in January, but that the differences became statistically significant 6 months later. As shown in Figure 5, the a* parameter decreased by about 2.0 units in both groups, suggesting that sun protection has little influence on this component.

\section{Impact of a 3-year period on skin aging}

The selection of the same group of Chinese women in the winters of both 2008 and 2011 allowed us to assess the possible interaction between a 3-year period of skin "aging" and skin tone, to determine more precisely the impact of daily sun protection on skin tone, and to analyze the present findings in light of our previous research. ${ }^{3,4}$ Figures $6-8$ indicate a slight but not statistically significant 0.1 unit decrease in $L^{*}$, a statistically significant increase in $\mathrm{b}^{*}(P=0.049)$, and a statistically significant 1.0 unit decrease in ITA $(P=0.04)$ between the winters of 2008 and 2011.

\section{Effects of sun protection versus age-related changes}

Figure 5 summarizes the apparent relative contributions of normal skin aging and sun protection in the same group of women. It appears that, over a period of 6 months from winter through summer, unprotected skin developed a darker tone (indicated by a significant 1.0 unit decrease in $\mathrm{L}^{*}$ ), whereas strongly sun-protected skin became lighter, albeit not to a degree that was statistically significant. The observed changes appeared to be positive (indicated by a gain in $\mathrm{L}^{*}$ ) for the group with sun protection in 2011 and negative (a decrease in $\mathrm{L}^{*}$ ) for the group without sun protection in 2008. This difference between the two groups was significantly different $(P<0.001)$.

\section{Discussion}

The present data confirm our earlier finding that the skin of Chinese women darkens over the 6-month period from winter through summer, ${ }^{3}$ a phenomenon referred to here as skin seasonal dark-

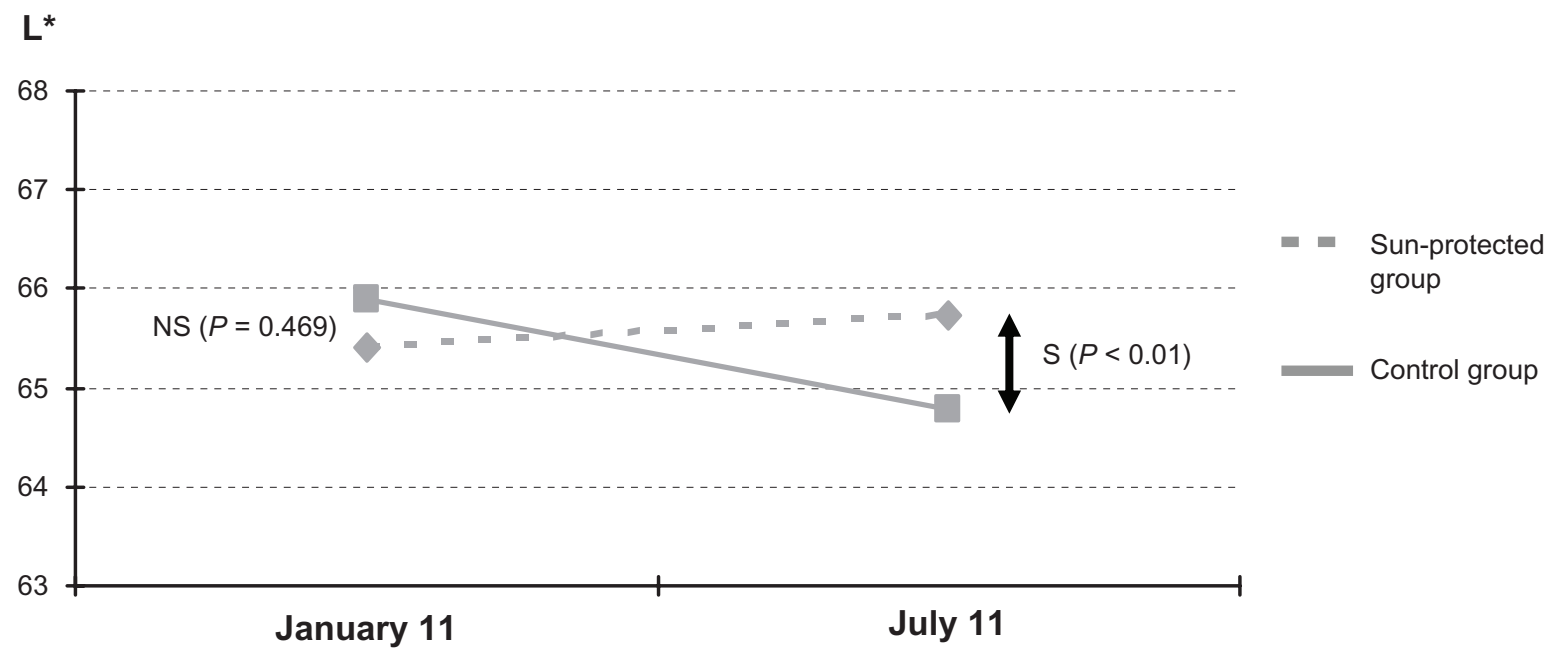

Figure I Changes in lightness $\left(L^{*}\right)$ from winter to summer in both groups, sun-protected group vs control group. Abbreviations: NS, not significant; S, significant. 


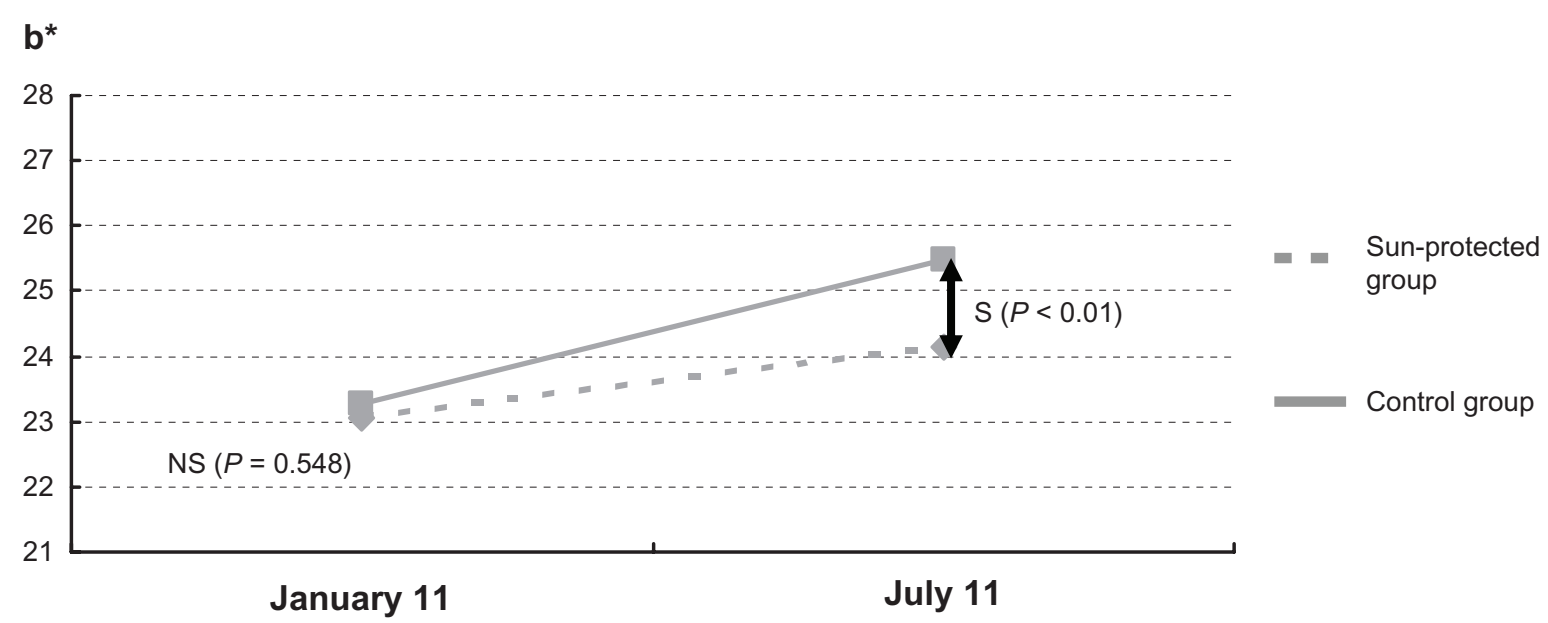

Figure 2 Changes in b* (yellowness) from winter to summer $201 \mathrm{I}$ in both groups, sun-protected group vs control group. Abbreviations: NS, not significant; S, significant.

ening. Importantly, this study also shows that regular application of a sun-protective cosmetic product counteracts this darkening process in Chinese women. To our knowledge, this study is the first to provide evidence of a positive role for a sunscreen-based product in mitigating or preventing these seasonally induced alterations in skin tone. As already shown by others, ${ }^{6-10}$ sun exposure is the leading factor in skin pigmentation changes in humans. In Shanghai, as in most parts of the world, except for equatorial regions, the passage from winter through summer results in increased daily light exposure as well as an increase in ultraviolet $\mathrm{A}$ and $\mathrm{B}$ radiation. ${ }^{11}$ Therefore, long-term protection against the detrimental effects of sunlight using a highly protective product appears to be a logical and efficient strategy.

In most cases, the parameters measured in this study varied significantly by several units in the control group as compared with the sun-protected group. However, it should be borne in mind that these parameters have a rather restricted range. For example, we previously found that the aging process in the 20-80-year age group produces a regular decrease in skin lightness $\left(\mathrm{L}^{*}\right)$ of about 1.0 unit per decade, and a decrease in ITA, a more dynamic parameter, by about 2.0-3.0 units in the same time interval. ${ }^{3,4}$ The drop of about 4.0 units in a 6-month period, which is comparable with the decrease we found previously in controls, represents an apparent paradox in relation to the expected steady 1.0 unit decrease every 4 years during aging, and suggests that the changes in skin tone seen from January through July are likely to be transient and reversed by $90 \%-95 \% 6$ months later, ie, that the process is possibly cyclic. Skin will darken when exposed to ultraviolet ray due to facultative pigmentation,

\section{ITA}

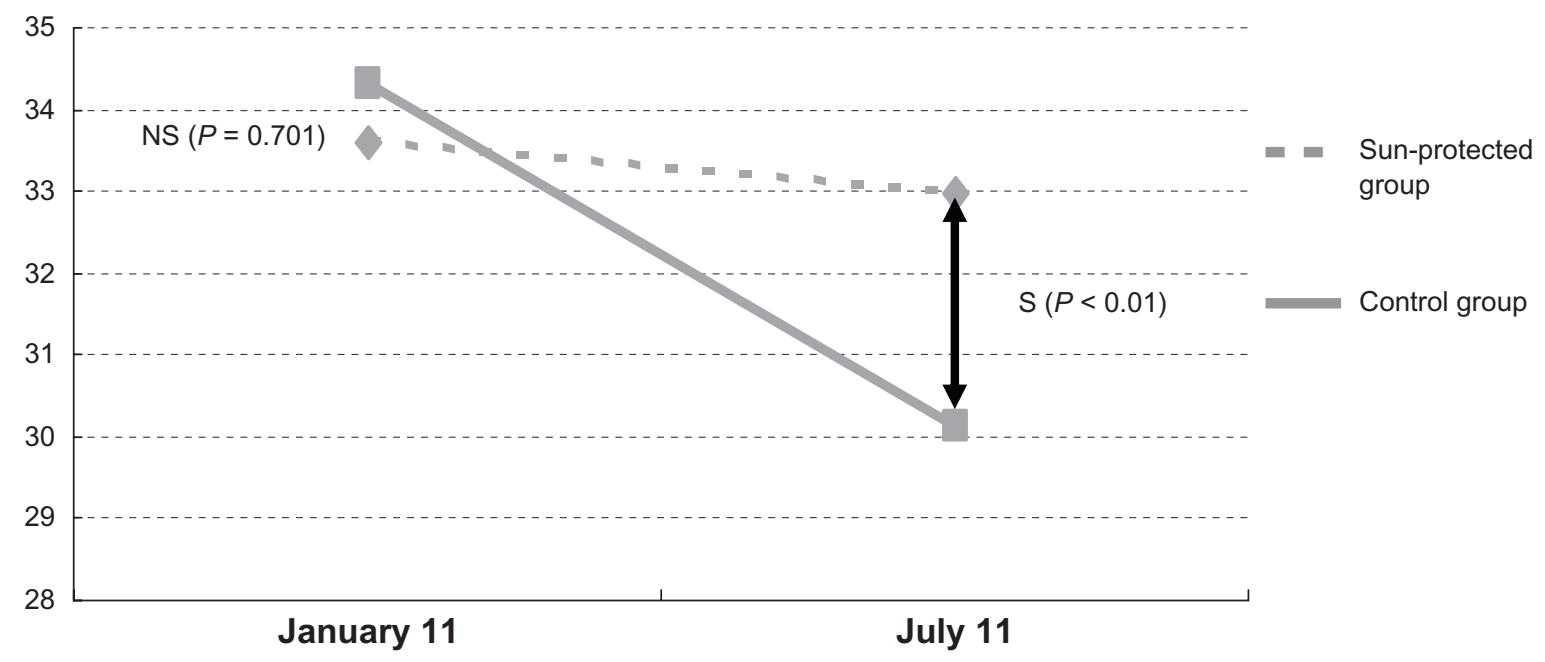

Figure 3 Changes in individual typological angle (ITA) from winter to summer $201 \mathrm{I}$ in both groups, sun-protected vs control group. Abbreviations: NS, not significant; S, significant. 


\section{A}

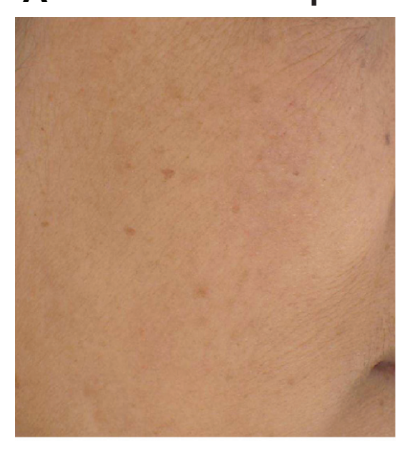

Base line (T0)

B

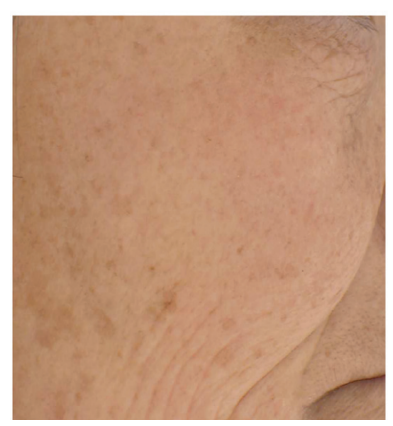

Base line (T0)

\section{Control group}

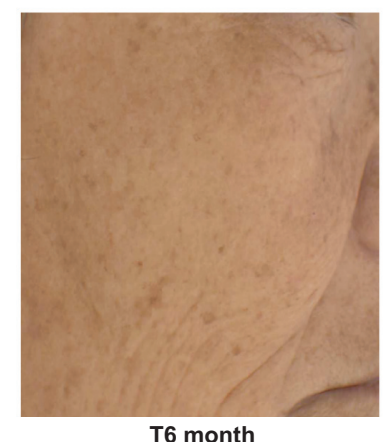

T6 month
Figure 4 (A) Chromasphere picture for efficacy demonstration with photoprotection. (B) Picture for efficacy demonstration without photoprotection. Note: T0 baseline meaning winter, T6 month meaning summer after 6 months.

but when the stimulus, ie, ultraviolet ray greatly decrease; the process will switch back from facultative to constitutive pigmentation. During this switch, because the sun protection required by the skin is minimal and the desquamation process is ongoing, the pigment accumulated over the summer months is lost and skin color returns close to normal. Along the same line, a small proportion $(5 \%-10 \%)$ of skin tone would be expected not to recover because of progressive accumulation of slight darkening over the years because of seasonal skin darkening.

From a technical point of view, the various parameters measured using the Chromasphere technique are highly reproducible. Apart from regular internal calibration of color standards, previous studies based on successive images of the same subjects at close intervals have shown high reproducibility of about \pm 0.1 unit in $\mathrm{L}^{*}$ despite various possible sources of bias, such as flushing brought on by a sudden surge of emotion or incorrect repositioning of the face towards the camera.

The sun-protected group did not show a statistically significant increase in $\mathrm{L}^{*}$, in contrast with the control group which showed a significant 1.0 unit decrease in $\mathrm{L}^{*}$ (Figure 1), which is consistent with our previous findings. ${ }^{4}$ Both groups showed an increase in $b^{*}$ (yellow-blue component), albeit to a lesser extent in the sun-protected group (Figure 2), of approximately 1.0 unit between January and July, with both groups showing a statistically significant increase in July. Bearing in mind that skin "yellowing" is a deeply anchored Chinese cultural feature ("old and married women turn yellow" according to a Chinese dictum), a sun-protective cosmetic product goes well beyond skin protection alone by mitigating changes in skin tone that have a profound social impact. The more dynamic ITA parameter confirms these observations, showing a 3.0 unit decrease in the control group versus

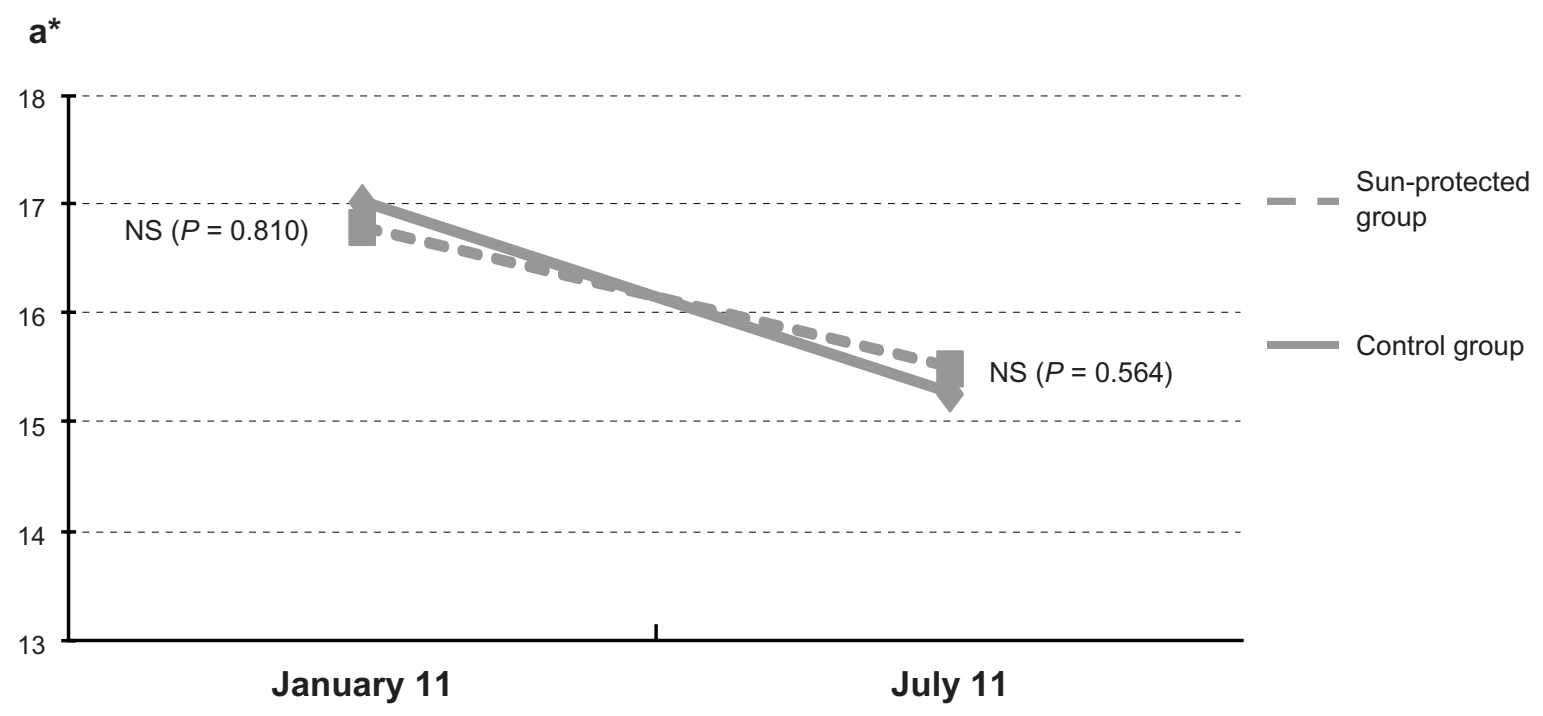

Figure 5 Changes in the red/green component $\left(\mathrm{a}^{*}\right)$ from winter to summer $20 \mathrm{II}$ in both groups, sun-protected vs control group. Abbreviations: NS, not significant; S, significant. 


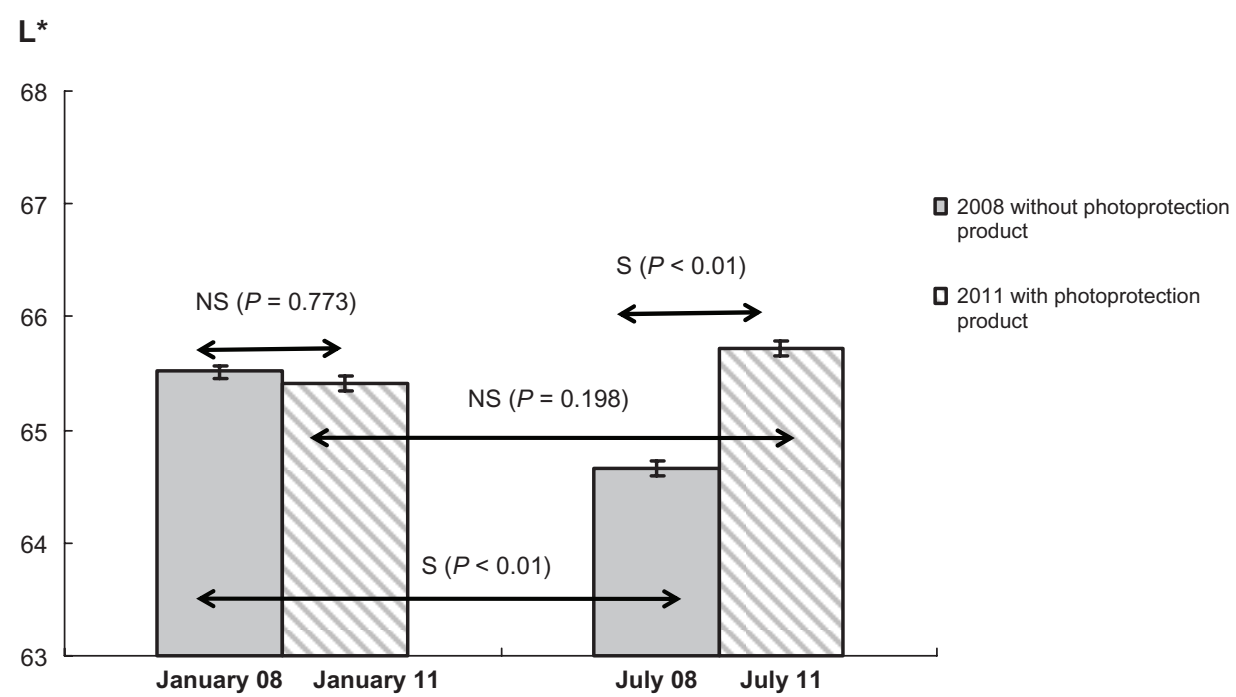

Figure 6 Changes in skin lightness ( $\left.L^{*}\right)$ from winters to summers 2008 and 201 I on the same group of women, with and without photoprotection product. Abbreviations: NS, not significant; S, significant.

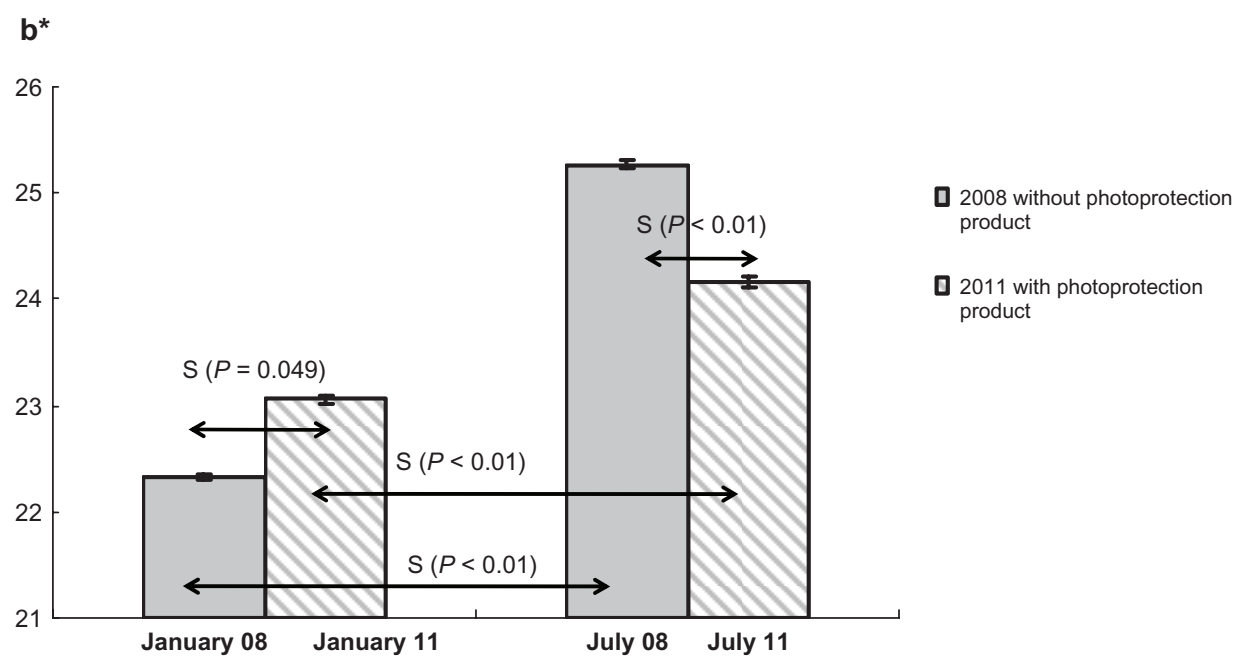

Figure 7 Changes in $b^{*}$ (yellowness) from winters to summers 2008 and 2011 on the same group of women, with and without photoprotection product. Abbreviations: NS, not significant; S, significant.

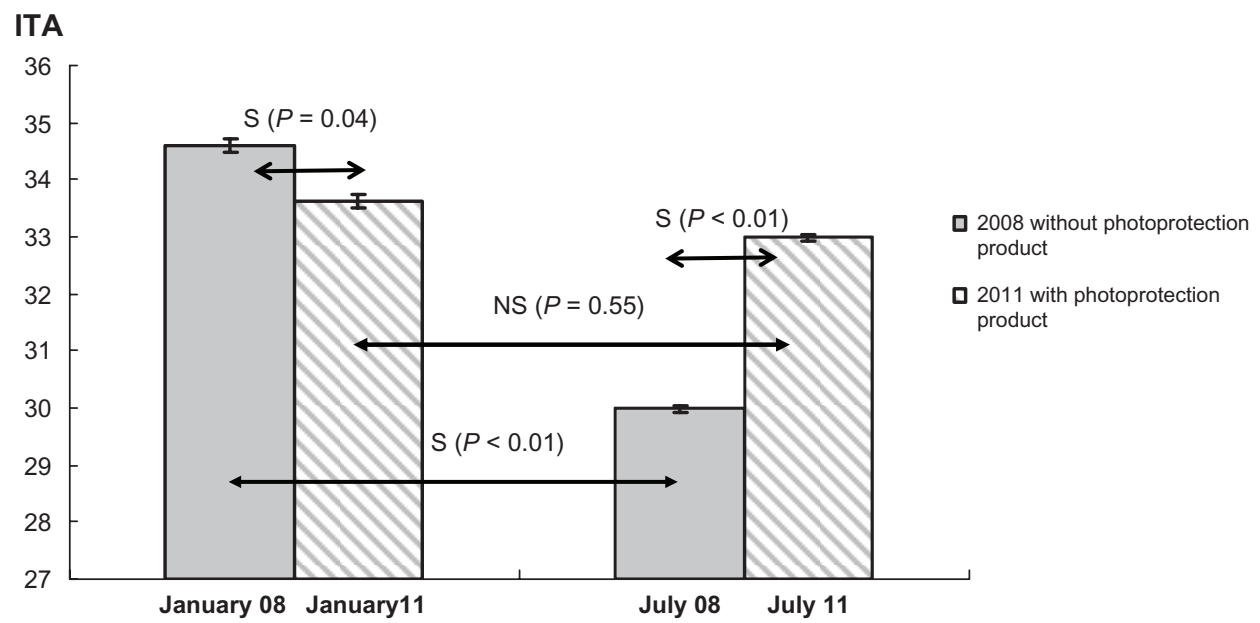

Figure 8 Changes in individual typological angle (ITA) from winters to summers 2008 and $201 \mathrm{I}$ on the same group of women, with and without photoprotection product. Abbreviations: NS, not significant; S, significant. 
a 1.0 unit decrease in the sun-protected group (Figure 3). The difference in ITA values between the two groups was statistically significant in July.

Our reproducible measurements showing changes in the various parameters of $0.5-3.0$ units as a result of sun protection over a 6-month period are important. For the present 2011 study, our results were obtained using a very strong protective product (SPF50+, PPD 18) which contained $0.2 \%$ vitamin $\mathrm{C}$ as an antioxidant. At such a low level, it is improbable that the product had a whitening action. ${ }^{12,13}$ From the data shown here, it cannot be concluded that a lower index of protection would have yielded comparable results. Hence, it would not be appropriate to compare our present findings with the results of our earlier research showing the effects of a combination of a whitening product and lower-level sun protection (SPF18, PA+++). ${ }^{3,4}$ In addition, two major relevant factors in this study could not be controlled for. First, the daily sun exposure of each subject could not be measured or estimated. Second, because application of the product was done at home, the actual amount of product applied is unknown. However, it seems likely that our subjects, like most consumers, ${ }^{14,15}$ did not apply the standard amount of $2 \mathrm{mg} / \mathrm{cm}^{2}\left(1.2 \mathrm{mg} / \mathrm{cm}^{2}\right.$ from normal data) to reach the true SPF50+ and IPA 18 of the product. Such limitations are well recognized by researchers when studying subjects who are at liberty to use a given product as part of their usual daily routine. Clearly, further work is needed to determine the minimal protection indices needed to bring about unchanged L* or ITA in comparable but controlled conditions.

Despite the above limitations, this study clearly shows that both seasonal skin darkening and yellowing in Chinese women can be largely mitigated by daily application of a sun-protective cosmetic product. Assuming that a 3-year period can represent an acceptable approach to monitoring the effects of aging on the skin, the same group of Chinese women examined in 2008 and 2011 allows some additional comments to be made. First, our research confirms the skin seasonal darkening previously observed from winter through summer 2008 in Chinese women. ${ }^{4}$ Second, comparing the same group in the winters of 2008 and 2011 enabled us to identify subtle changes related to "aging" during this 3-year interval. In our earlier study in 2008, normal skin aging was associated with a steady 1.0 unit decrease in $\mathrm{L}^{*}$ and a 2.5 unit decrease in ITA per decade (ie, one ITA unit every 4 years) on average, whereas a* and $b^{*}$ showed a very slight increase. Three years later, this trend was confirmed, at least for $\mathrm{L}^{*}$ and ITA, with both parameters showing a 1.0 unit decrease. In terms of slope per year, these results appear consistent with our previous findings, suggesting that repeated seasonal skin darkening is a cumulative process in the overall skin darkening that commonly occurs in Chinese women.

The present study confirms that seasonal skin darkening does occur in Chinese women and, for the first time, shows that this phenomenon can be largely mitigated or prevented by daily use of a strong sun-protective cosmetic product. Taken together, the data indirectly validate the widely accepted view that ultraviolet radiation exposure is a leading contributor to the changes in skin appearance, including color, that occur in normal healthy skin.

\section{Disclosure}

The authors report no conflicts of interest in this work.

\section{References}

1. Wulf HC, Sandby-Møller J, Kobayasi T, Gniadecki R. Skin aging and natural photoprotection. Micron. 2004;35:185-191.

2. Nouveau-Richard S, Yang Z, Mac-Mary S, et al. Skin ageing: a comparison between Chinese and European populations, a pilot study. J Dermatol Sci. 2005;40:187-193.

3. Qiu HX, Long XH, Flament F. Influence of season on some skin properties: winter vs summer, as experienced by 354 Shanghaiese women of various ages. Int $J$ Cosmet Sci. 2011;33:377-383.

4. Qiu HX, Long XH, Flament F. Instrumental and clinical studies of the facial skin tone and pigmentation of Shanghaiese women. Changes induced by age and a cosmetic whitening product. Int J Cosmet Sci. 2012;34:49-54.

5. De Rigal J, Abella ML, Giron F, Caisey L, Lefebvre MA. Development and validation of a new skin color chart. Skin Res Technol. 2007;13: 101-109.

6. Sturm RA. Human pigmentation genes and their response to solar UV radiation. Mutat Res. 1998;422:69-76.

7. Jablonski NG, Chaplin G. The evolution of human skin coloration. J Hum Evol. 2000;39:57-106.

8. Rees JL. The genetics of sun sensitivity in humans. Am J Hum Genet. 2004;75:739-751.

9. Madhu A, Pathak MB, Dan L. Photobiology of melanin pigmentation: dose/response of skin to sunlight and its contents. JAm Acad Dermatol. 1983;9:724-733.

10. Madhu A, Pathak MB. Sunscreens: topical and systemic approaches for protection of human skin against harmful effects of solar radiation. J Am Acad Dermatol. 1982;7:285-312.

11. Dvorkin AY, Steinberger EH. Modeling the altitude effect on solar UV radiation. Solar Energy. 1999;65:181-187.

12. Bendich A, Machlin LJ, Scandurra O, Burton GW, Wayner DDM. The antioxidant role of vitamin C. Advances in Free Radical Biology and Medicine. 1986;2:419-444.

13. Shapiro SS, Saliou C. Role of vitamins in skin care. Nutrition. 2001;17: 839-844.

14. Diffey B. Sunscreen isn't enough. J Photochem Photobiol B. 2001;64: $105-108$.

15. McLean DI, Gallagher R. Sunscreens use and misuse. Dermatol Clin. 1998;16:219-226. 


\section{Publish your work in this journal}

Clinical, Cosmetic and Investigational Dermatology is an international, peer-reviewed, open access, online journal that focuses on the latest clinical and experimental research in all aspects of skin disease and cosmetic interventions. All areas of dermatology will be covered; contributions will be welcomed from all clinicians and

basic science researchers globally. This journal is indexed on CAS.

The manuscript management system is completely online and includes

a very quick and fair peer-review system, which is all easy to use. Visit http://www.dovepress.com/testimonials.php to read real quotes from published authors.

Submit your manuscript here: http://www.dovepress.com/clinical-cosmetic-and-investigational-dermatology-journal 